\title{
Resposta à carta ao editor referente ao artigo: Fixação intramedular flexível para fraturas diafisárias dos ossos do antebraço em crianças
}

\section{Reply to Letter to Editor Regarding the Article: Intramedullary Flexible Nailing for the Diaphyseal Fractures of Forearm Bones in Children}

\author{
Balakrishnan M. Acharya ${ }^{10}$ Pramod Devkota10 \\ ${ }^{1}$ Departamento de Ortopedia e Cirurgia de Trauma, Academia Patan \\ de Ciências da Saúde, Hospital Patan, Lalitpur, Nepal \\ Rev Bras Ortop 2020;55(6):809.
}

\author{
Abhishek K. Thakur $^{1 \oplus}$ Bidur Gyawali $^{1 \oplus}$
}

Embora as fraturas dos ossos do antebraço sejam tratadas com sucesso de forma conservadora, os desfechos permanecem variáveis e, posteriormente, alguns casos podem exigir manipulação adicional de fraturas ou intervenção cirúrgica formal devido a angulações residuais. ${ }^{1}$ Estudos anteriores mostraram que a falha no tratamento não operatório de fraturas de entre-eixos em populações pediátricas varia entre $39 \%$ e $64 \%{ }^{2}$

Atualmente, as demandas do mundo moderno nos levam a considerar as dificuldades do tratamento não cirúrgico, mantendo as crianças em gesso. Os pais trabalham, o atendimento domiciliar é difícil, o tempo longe da escola e até mesmo questões de conforto do paciente são considerados. Portanto, as fraturas, que costumavam ser tratadas de modo não cirúrgico anteriormente, passaram a ser tratadas cirurgicamente, com abordagem sem sangue, nos dias de hoje. ${ }^{3}$

Nos últimos tempos, o uso de haste intramedular (IM) flexível tem sido amplamente realizado para fraturas no antebraço pediátrico devido às vantagens da técnica minimamente invasiva e da prevenção de complicações relacionadas aos pinos, que mudou os princípios tradicionais do cuidado com a fratura do antebraço pediátrico. ${ }^{4}$ Peterlein et al relataram bons resultados em longo prazo de fraturas no antebraço pediátrico tratadas com haste intramedular. ${ }^{5}$ Martus et al concluíram que a técnica de procedimento com a haste
Endereço para correspondência Pramod Devkota, MBBS MS(Ortho), Departamento de Ortopedia e Cirurgia de Trauma, Academia Patan de Ciências da Saúde, Patan Hospital, Lalitpur, Nepal (e-mail: devkotap@gmail.com).

intramedular é eficaz para fraturas no antebraço pediátrico com resultados bons ou excelentes em $91 \%$ dos casos. $^{6}$

\section{Referências}

1 Reinhardt KR, Feldman DS, Green DW, Sala DA, Widmann RF, Scher DM. Comparison of intramedullary nailing to plating for both-bone forearm fractures in older children. J Pediatr Orthop 2008;28(04):403-409

2 Bowman EN, Mehlman CT, Lindsell CJ, Tamai J. Nonoperative treatment of both-bone forearm shaft fractures in children: predictors of early radiographic failure. J Pediatr Orthop 2011; 31(01):23-32

3 Alvachian Fernandes HJ, Saad EA, Reis FB. Osteosynthesis with intramedullary nails in children. Rev Bras Ortop 2015;44(05): 380-385

4 Kim BS, Lee YS, Park SY, Nho JH, Lee SG, Kim YH. Flexible Intramedullary Nailing of Forearm Fractures at the Distal Metadiaphyseal Junction in Adolescents. Clin Orthop Surg 2017;9(01): 101-108

5 Peterlein CD, Modzel T, Hagen L, Ruchholtz S, Krüger A. Long-term results of elastic-stable intramedullary nailing (ESIN) of diaphyseal forearm fractures in children. Medicine (Baltimore) 2019;98 (11):e14743

6 Martus JE, Preston RK, Schoenecker JG, Lovejoy SA, Green NE, Mencio GA. Complications and outcomes of diaphyseal forearm fracture intramedullary nailing: a comparison of pediatric and adolescent age groups. J Pediatr Orthop 2013; 33(06):598-607
DOI https://doi.org/ 10.1055/s-0040-1712140. ISSN 0102-3616.
Copyright $\odot 2020$ by Sociedade Brasileira License terms de Ortopedia e Traumatologia. Published by Thieme Revinter Publicações Ltda, Rio de Janeiro, Brazil 